\title{
APPROACH TO EQUILIBRIUM FOR A FORCED BURGERS EQUATION
}

\author{
WERNER KIRSCH AND BARRY SIMON ${ }^{1}$
}

\begin{abstract}
We show that approach to equilibrium in certain forced Burgers equations is implied by a decay estimate on a suitable intrinsic semigroup estimate, and we verify this estimate in a variety of cases including a periodic force.
\end{abstract}

\section{INTRODUCTION}

This paper is a contribution to the literature [10, 11, 3] on large time asymptotics of the forced Burgers equation

$$
\frac{\partial u_{i}}{\partial t}+\sum_{j=1}^{\nu} u_{j} \frac{\partial u_{j}}{\partial x_{i}}=\frac{1}{2} \Delta u_{i}+\frac{\partial V(x)}{\partial x_{i}}, \quad t \geq 0, x \in \mathbb{R}^{\nu}, i=1, \ldots, \nu
$$

where $u$ is real valued. We will make two assumptions on the initial data $u_{j}(x, t=0)=u_{j}^{(0)}(x)$ :

$$
\begin{aligned}
& \text { (i) } \frac{\partial u_{i}^{(0)}}{\partial x_{j}}=\frac{\partial u_{j}^{(0)}}{\partial x_{i}} \quad \text { all } i, j \\
& \text { (ii) } \quad \psi_{0}(x) \equiv \int_{0}^{x} \vec{u}^{(0)}(y) \cdot d y \in L^{\infty} .
\end{aligned}
$$

(1.2), which is vacuous in the standard $\nu=1$ case, implies that the value of $\psi_{0}$ given by (1.3) is independent of the path taken from 0 to $x$ in the line integral. Typical of our results is:

Theorem 1.1. Let $V$ be a $C^{1}$ periodic function on $\mathbb{R}^{\nu}$. Then, there is a unique initial condition $u_{\infty}^{(0)}(x)$ obeying (1.2), (1.3) for (1.1) whose solution is independent of $t$. Moreover, if $u^{(0)}$ is any other initial data obeying $(1.2) /(1.3)$, then

$$
\lim _{t \rightarrow \infty} \sup _{x}\left[\left|u(x, t)-u_{\infty}^{(0)}(x)\right|\right]=0 .
$$

\footnotetext{
${ }^{1}$ Supported in part by NSF Grant No. DMS-9707661.

Date: June 11, 2001.

* To appear in Journal of Evolution Equations.
} 
Remark. (1.3) does not imply that $u^{(0)}$ is $L^{\infty}$, but our proof shows that for $t>0, u(\cdot, t) \in L^{\infty}$. Thus, for $t>0$, the quantity in the limit in (1.4) is finite.

What is new about our ideas and Theorem 1.1 is that there is no regularity condition on the initial condition $u$ at infinity other than (1.3). Previous approaches require at least that $e^{-\psi_{0}}$ have some kind of average. To understand how we overcome this, we need to begin the proof by reminding the reader of the Cole-Hopf transformation. Define

$$
\varphi_{0}(x)=\exp \left(-\psi_{0}(x)\right)
$$

so that

$$
u_{i}^{(0)}=-\varphi_{0}^{-1} \frac{\partial \varphi_{0}}{\partial x_{i}} .
$$

$V$ is bounded so

$$
H=-\frac{1}{2} \Delta+V
$$

is bounded below. Thus we add a constant to $V$ so that henceforth

$$
\inf \operatorname{spec}(H)=0 \text {. }
$$

Define

$$
\varphi(x, t)=\left(e^{-t H} \varphi_{0}\right)(x) .
$$

Then direct manipulation shows that

Proposition 1.2. Let $\psi_{0} \in L^{\infty}$ be $C^{1}$ and let $\varphi_{0}, \varphi$ obey (1.5), (1.9). Then, for $t>0, \varphi(x, t)>0$ and $\varphi, \nabla \varphi, \Delta \varphi$ are $C^{1}$ and

$$
u(x, t)=-\varphi(x, t)^{-1}(\nabla \varphi)(x, t)
$$

obeys (1.1) for $t>0$ and $\lim _{t \downarrow 0} u(\cdot, t)=u^{(0)} \equiv \nabla \psi_{0}$.

Remarks. 1. It follows from [9] that with $V$ a $C^{1}$ function with bounded derivatives that $\varphi$ and $(-\Delta+V) \varphi$ are $C^{1}$. It follows that $\Delta \varphi$ is $C^{1}$ which by elliptic regularity means $\nabla \varphi$ is $C^{1}$. The Laplacian here and in (1.1) may be distributional rather than classical. If $\nabla V$ is assumed Hölder continuous, we can replace these by classical derivatives.

2. As we will discuss below, $e^{-t H}$ maps $L^{\infty}$ to $L^{\infty}$ and (1.9) is intended in the sense of the $L^{\infty}$ map.

3. This result does not require that $V$ be periodic; $V$ need only be $C^{1}$ and in $L^{\infty}$. We will use it in this form below.

When $V$ is periodic, it has a periodic ground state $\Omega$, that is, a positive periodic solution of

$$
\left(-\frac{1}{2} \Delta+V\right) \Omega=0 .
$$


Thus

$$
u_{\infty}^{(0)}(x)=-\Omega(x)^{-1}(\nabla \Omega)(x)
$$

is a stationary solution of (1.1). A natural approach to (1.4) is to prove that

$$
\varphi \rightarrow c \Omega
$$

and

$$
\nabla \varphi \rightarrow c \nabla \Omega
$$

both in $L^{\infty}$. This is essentially what previous works do.

To understand the limitations of this approach and why one can hope to go beyond them, consider the case $V \equiv 0$. Then $u_{\infty}=0, \Omega \equiv 1$, and

$$
\varphi(x, t)=(2 \pi t)^{-\nu / 2} \int \exp \left(-(x-y)^{2} / 2 t\right) \varphi_{0}(y) d y .
$$

If $(2 R)^{-\nu} \int_{\sup _{i}\left|y_{i}\right| \leq R} \varphi_{0}(y) d^{\nu} y \rightarrow c$ as $R \rightarrow \infty$, it is not hard to see that $\varphi(x, t) \rightarrow c$ as $t \rightarrow \infty$ for each fixed $x$, so (1.13) holds, but this is not true in general.

For example, if $R_{n}=e^{e^{n}}$ and

$$
\varphi_{0}(y)=2+(-1)^{n} \quad \text { if } \quad R_{n}<\sup _{i}\left|y_{i}\right|<R_{n+1}
$$

then for $t \sim R_{n} R_{n+1}$, it is not hard to see that $\varphi(0, t) \sim 2+(-1)^{n}$ and thus $\varphi(0, t)$ does not have a limit. But in this example, one can see that $\nabla \varphi$ does go to zero.

Our key observations are that rather than prove (1.13) and (1.14) separately, it suffices to prove that

$$
\nabla(\varphi / \Omega) \rightarrow 0
$$

and that (1.15) is equivalent to some estimates on the intrinsic semigroup associated to $H$. Specifically, let $K_{t}(x, y)$ be the integral kernel of $e^{-t H}$ and let

$$
L_{t}(x, y)=\Omega^{-1}(x) K_{t}(x, y) \Omega(y)^{-1}
$$

$L_{t}$ is the kernel of a semigroup on $L^{2}\left(\mathbb{R}^{\nu}, \Omega^{2} d^{\nu} x\right)$.

To prove Theorem 1.1, we will prove two estimates:

$$
\left|\partial_{x} L_{t}(x, y)\right| \leq C t^{-\nu / 2}\left[\exp \left(-D(x-y)^{2} / t\right)+\exp (-E|x-y|)\right]
$$

for suitable $C, D$ and all $t>1$, all $x, y$ and

$$
\left|\partial_{x} L_{t}(x, y)\right| \leq C t^{-(\nu+1) / 2}
$$

all $t>1$, all $x, y$. 
We will show that if $\Omega$ obeys $0<a \leq \Omega<b$, is $C^{3}$ and $V \equiv$ $\frac{1}{2} \Omega^{-1}(\Delta \Omega)$ is bounded and uniformly Hölder continuous, then (even if $V$ is not periodic)

$$
\left|\partial_{x} L_{t}(x, y)\right| \leq C t^{-\alpha}\left[\exp \left(-D(x-y)^{2} / t\right)\right]
$$

for some $\alpha>\nu / 2$, all $t \geq 1$. This will lead to the following generalization of Theorem 1.1:

Theorem 1.3. Let $V$ be a $C^{1}$ function and suppose that $V$ is bounded and uniformly Hölder continuous. Suppose that $-\frac{1}{2} \Delta+V$ has a ground state $\Omega$ obeying $0<a \leq \Omega \leq b$ for some $a, b$ and all $t$. Then there is a unique initial condition $u_{\infty}^{(0)}(x)(=\nabla \Omega / \Omega)$ obeying (1.2), (1.3) for (1.1) whose solution is independent of $t$. Moreover, if $u^{(0)}$ is any other initial data obeying (1.2)/(1.3), then

$$
\lim _{t \rightarrow \infty} \sup _{x}\left[\left|u(x, t)-u_{\infty}^{(0)}(x)\right|\right]=0 .
$$

Certain quasiperiodic Schrödinger operators have a quasiperiodic ground state [5]. Thus the above theorem applies to this situation as well; see 11$]$.

In Section 2, we will reduce Theorems 1.1 and 1.3 to (1.19). In Section 3, we will derive (1.17) using ideas due to Davies. In Section \&, we will prove (1.18) in the periodic case and (1.19) in general.

We are dedicating this paper to the memory of Tosio Kato, who taught us so much about Schrödinger operators, about semigroups, and about non-linear equations, areas which come together here.

\section{Reduction to Intrinsic Heat Kernel Estimates}

According to Proposition 1.2, the solution $u$ of (1.1) is related to a solution of (1.9) via (1.16). This leads to

Theorem 2.1. Suppose there exist $\Omega$ so

$$
e^{-t H} \Omega=\Omega
$$

and

$$
0<a \leq \Omega(x) \leq b
$$

and that

$$
\lim _{t \rightarrow \infty}\|\vec{\nabla}(\varphi(\cdot, t) / \Omega(\cdot))\|_{\infty}=0 .
$$

Then $(\overline{1.20})$ holds with $u_{\infty}^{(0)}=\nabla \Omega / \Omega$. 
Proof. By (1.3), we have

$$
0<c_{1} \leq \varphi_{0} \leq c_{2}
$$

so by $(2.2)$

$$
c_{1} b^{-1} \Omega \leq \varphi_{0} \leq c_{2} a^{-1} \Omega .
$$

Since $e^{-t H}$ is positivity preserving and (2.1) holds,

$$
c_{1} b^{-1} \Omega(x) \leq \varphi(x, t) \leq c_{2} a^{-1} \Omega(x) .
$$

So by (2.2) again,

$$
c_{1} b^{-1} a \leq \varphi(x, t) \leq c_{2} a^{-1} b
$$

Now

$$
\begin{aligned}
u(\cdot, t)-u_{\infty}^{(0)} & =(\nabla \varphi)(\cdot, t) / \varphi(\cdot, t)-(\nabla \Omega)(\cdot) / \Omega(x) \\
& =(\Omega \nabla \varphi-\varphi \nabla \Omega) / \varphi \Omega \\
& =[\nabla(\varphi(\cdot, t) / \Omega)][\Omega / \varphi] .
\end{aligned}
$$

Since $\varphi$ and $\Omega$ are uniformly in $t$ and $x$ bounded above and below, we see that $(\overline{1.20})$ is equivalent to $(2.3)$.

Now consider the unitary map $U: L^{2}\left(\mathbb{R}^{\nu}\right) \rightarrow L\left(\mathbb{R}^{\nu}, \Omega^{2} d x\right)$ by $(U f)(x)=$ $f(x) \Omega(x)^{-1}$ and let $M$ be the self-adjoint operator $U H U^{-1}$ on $L^{2}\left(\mathbb{R}^{\nu}, \Omega^{2} d x\right)$. Then, as is well-known (and a direct calculation),

$$
(f, M f)_{L^{2}\left(\mathbb{R}^{\nu}, \Omega^{2} d x\right)}=\int(\nabla f)^{2} \Omega^{2} d x
$$

or equivalently,

$$
M f=-\Delta f-2(\vec{\nabla} \Omega) \Omega^{-1} \cdot \vec{\nabla} f .
$$

Now let $K_{t}(x, y)$ be the integral kernel of $e^{-t H}$, that is,

$$
\left(e^{-t H} f\right)(x)=\int K_{t}(x, y) f(y) d^{\nu} y
$$

and let $L_{t}(x, y)$ be the integral kernel of $e^{-t M}$, that is,

$$
\left(e^{-t M} f\right)(x)=\int L_{t}(x, y) f(y) \Omega^{2}(y) d^{\nu} y .
$$

Since $e^{-t M}=U e^{-t H} U^{-1}$, we see that $L_{t}$ and $K_{t}$ are related by (1.16).

Now if $\varphi=e^{-t H} \varphi_{0}$, then

$$
\begin{aligned}
{[\varphi(\cdot, t) / \Omega(\cdot)] } & =U e^{-t H} \varphi_{0}=e^{-t M} U \varphi_{0} \\
& =\int L_{t}(\cdot, y) \varphi_{0}(y) \Omega(y) d^{\nu} y .
\end{aligned}
$$


Since $\varphi_{0}$ and $\Omega$ are uniformly bounded, we see that

$$
|\nabla[\varphi(x, t) / \Omega(x)]| \leq c \int\left|\partial_{x} L_{t}(x, y)\right| d^{\nu} y
$$

Thus:

Proposition 2.2. A sufficient condition for (2.3) to hold for any initial $\varphi_{0}$ (coming from a $u_{i}^{(0)}$ obeying $\left.(1.2) /(1.3)\right)$ is that

$$
\sup _{x} \int\left|\partial_{x} L_{t}(x, y)\right| d^{\nu} y \rightarrow 0
$$

as $t \rightarrow \infty$.

Theorem 2.3. If (1.19) holds or if (1.17)/(1.18) hold, then (2.6) holds.

Proof. (1.19) plus scaling implies that

$$
\int\left|\partial_{x} L_{t}(x, y)\right| d^{\nu} y \leq C_{1} t^{-\alpha} t^{\nu / 2}
$$

which goes to zero if $t \rightarrow \infty$ since $\alpha>\nu / 2$. (1.17)/(1.18) imply

$$
\left|\partial_{x} L_{t}(x, y)\right| \leq C t^{-\nu / 2-1 / 4}\left[\exp \left(-D(x-y)^{2} / 2 t\right)+\exp \left(-\frac{1}{2} E|x-y|\right)\right]
$$

which implies

$$
\int\left|\partial_{x} L_{t}(x, y)\right| d^{\nu} y \leq C_{1} t^{-1 / 4} \pm C_{2} t^{-\nu / 2-1 / 4}
$$

which goes to zero as $t \rightarrow \infty$.

\section{Exponential-Gaussian Estimates on $\partial_{x} L_{t}$}

Our goal in this section is to explain how one can get (1.17) from ideas of Davies [1, 2]. His ideas immediately imply an estimate

$$
\left|K_{t}(x, y)\right| \leq C_{\varepsilon} t^{-\nu / 2} \exp \left(-(x-y)^{2} /(4+\varepsilon) t\right)
$$

for any $\varepsilon>0$. (3.1) implies (even dropping the Gaussian) that

$$
\left\|e^{-t H}\right\|_{L^{1} \rightarrow L^{\infty}} \leq C t^{-\nu / 2}
$$

so by interpolation with boundedness on $L^{\infty}$ (see Simon [9] and references therein),

$$
\left\|e^{-t H}\right\|_{L^{1} \rightarrow L^{2}}=\left\|e^{-t H}\right\|_{L^{2} \rightarrow L^{\infty}} \leq C t^{-\nu / 4}
$$

Thus

$$
\left\|e^{-(t+i s) H}\right\|_{L^{1} \rightarrow L^{\infty}} \leq\left\|e^{-t H / 2}\right\|_{L^{2} \rightarrow L^{\infty}}\left\|e^{-i s H}\right\|_{L^{2} \rightarrow L^{2}}\left\|e^{-t H / 2}\right\|_{L^{1} \rightarrow L^{2}}
$$




$$
\leq C t^{-\nu / 2}
$$

and thus for any $|\theta|<\pi / 2$,

$$
\left\|\exp \left(-t e^{i \theta} H\right)\right\|_{L^{1} \rightarrow L^{\infty}} \leq C_{\theta} t^{-\nu / 2}
$$

which yields

$$
\left|K_{t e^{i \theta}}(x, y)\right| \leq C_{\theta} t^{-\nu / 2}
$$

By interpolation between (3.1) and (3.2), we see that for complex $t$ in a section $S_{\theta}=\{t|| \operatorname{Arg}(t) \mid \leq \theta\}$ we have if $\theta<\pi / 2$,

$$
\left|K_{t}(x, y)\right| \leq C_{\theta}|t|^{-\nu / 2} \exp \left(-D_{\theta}(x-y)^{2} /|t|\right)
$$

and this implies by a Cauchy estimate that in the same sectors

$$
\left|\partial_{t} K_{t}(x, y)\right| \leq C_{\theta}|t|^{-\nu / 2-1} \exp \left(-D_{\theta}(x-y)^{2} /|t|\right)
$$

(where $C_{\theta}, D_{\theta}$ can change value from one equation to the next).

Thus for $t \geq 1$ and real,

$$
\left|(-\Delta+V)_{x} K_{t}(x, y)\right| \leq C|t|^{-\nu / 2-1} \exp \left(-D(x-y)^{2} /|t|\right) .
$$

Since $V$ is bounded, (3.3) and (3.4) imply that for $t \geq 1$,

$$
\left|\left(-\Delta_{x}+1\right) K_{t}(x, y)\right| \leq C|t|^{-\nu / 2} \exp \left(-D(x-y)^{2} /|t|\right) .
$$

But $\partial_{x}\left(-\Delta_{x}+1\right)^{-1}$ has an explicit convolution integral kernel which is $L^{1}$ at short distances and exponentially decaying at large. This implies for $t \geq 1$

$$
\left|\left(\partial_{x} K_{t}\right)(x, y)\right| \leq C t^{-\nu / 2}\left[\exp \left(-D(x-y)^{2} / t\right)+\exp (-E|x-y|)\right]
$$

which, with (3.3) and the formula (1.16), implies (1.17). We summarize:

Theorem 3.1. The estimate (1.17) holds for any potential $V$ obeying the hypothesis of Theorem 1.3.

\section{Improved Time Decay Estimates on $\partial_{t} L_{t}$}

Here the goal is to show that

$$
\left|\left(\partial_{x} L_{t}\right)(x, y)\right| \leq C t^{-\alpha}
$$

for some $\alpha>\nu / 2$. We believe the estimate holds with $\alpha=\nu / 2+1 / 2$ and have proven this if $V$ is periodic: One makes a Bloch wave decomposition [8] to write the semigroup as an integral over the Brillouin zone and a Gaussian approximation to control the resulting integral. One uses the fact that the minimum of the bottom band is known to be a unique point with a strictly quadratic minimum [4. In general, 
we rely on estimates of Porper-Eidel'man [6] and only get $\alpha>\nu / 2$. (In a later paper [7], they get $\alpha=\nu / 2+1 / 2$ for the case $p=1$ below that does not accommodate our situation.)

Indeed, (4.1) is exactly Corollary 3.4 of their paper which they prove for fundamental solutions of equations of the form

$$
p(x) \partial_{t} u=\nabla \cdot(a(t, x) \nabla u),
$$

where $p, a$ and $\nabla a$ are all Hölder continuous.

But (2.5) can be rewritten

$$
M f=-\Omega^{-2} \nabla \cdot\left(\Omega^{2} \nabla f\right)
$$

So

$$
\partial_{t} u=-M u
$$

is of the form $(4.2)$ where

$$
p=\Omega^{2} \quad \text { and } \quad a_{i j}=\Omega^{2} \delta_{i j} .
$$

Thus, their result applies so long as $\Omega$ is $C^{1}$ with $\nabla \Omega$ uniformly Hölder continuous.

Since $\Delta \Omega=V \Omega$ with $V \in C^{1}$ and $\Omega$ a priori bounded, we see that

$$
\nabla \Omega=\nabla(-\Delta+1)^{-1}(-V+1) \Omega .
$$

Since $(-V+1) \Omega$ is bounded, the explicit integral kernel for $\nabla(-\Delta+1)^{-1}$ shows $\nabla \Omega$ is uniformly Hölder continuous of any order less than 1 . Thus, their Corollary 3.4 applies and (4.1) holds.

Acknowledgments. W.K. would like to thank T. Tombrello for the hospitality of Caltech where this research was done. We would like to thank E.B. Davies, T. Coulhon, A. Grigoryan, Y. Pinchover, and D. Robinson for valuable correspondence.

\section{REFERENCES}

[1] E.B. Davies, Explicit constants for Gaussian upper bounds on heat kernels, Amer. J. Math. 109 (1987), 319-334.

[2] E.B. Davies, Heat Kernels and Spectral Theory, Cambridge Tracts in Mathematics 92, Cambridge University Press, Cambridge, 1989.

[3] W. Kirsch and A. Kutzelnigg, Time asymptotics for solutions of the Burgers equation with a periodic force, Math. Z. 232 (1999), no. 4, 691-705.

[4] W. Kirsch and B. Simon, Comparison theorems for the gap of Schrödinger operators, J. Funct. Anal. 75 (1987), 396-410.

[5] S.M. Kozlov, Ground states of quasiperiodic operators, Sov. Math. Dokl. 28 (1983), 114-119. Translated from Dokl. Akad. Nauk SSSR 271 (1983), 532536. 
[6] F.O. Porper and S.D. Eidel'man, Two-sided estimates of fundamental solutions of second-order parabolic equations, and some applications, Russian Math. Surveys 39 (1984), no. 3, 119-178. Translated from Usp. Mat. Nauk. 39 (1984), no. 3, 107-156.

[7] F.O. Porper and S.D. Eidel'man, Theorems related to the asymptotic proximity of fundamental solutions to parabolic equations, Dokl. Math. 52 (1995), no. 2, 237-240. Translated from Dokl. Akad. Nauk 344 (1995), no. 5, 586-589.

[8] M. Reed and B. Simon, Methods of Modern Mathematical Physics, IV. Analysis of Operators, Academic Press, New York, 1978.

[9] B. Simon, Schrödinger semigroups, Bull. Amer. Math. Soc. 7 (1982), 447-526.

[10] Ya.G. Sinai, Two results concerning asymptotic behavior of solutions of the Burgers equation with force, J. Stat. Phys. 64 (1991), no. 1-2, 1-12.

[11] Ya.G. Sinai, Asymptotic behavior of solutions of $1 D$-Burgers equation with quasi-periodic forcing, Topol. Methods Nonlinear Anal. 11 (1998), no. 2, 219226.

W. Kirsch, Institut für Mathematik, Ruhr-Universität Bochum, D44780 Bochum, Germany

E-mail address: werner@mathphys.ruhr-uni-bochum.de

B. Simon, Division of Physics, Mathematics, and Astronomy, 253-37, California Institute of Technology, Pasadena, CA 91125-3700, USA

E-mail address: bsimon@caltech.edu 